\title{
Busca de Tratamento por Portadores de Tuberculose Pulmonar: Estudo Qualitativo Baseado no Modelo "Comportamento de Enfermo"
}

The Search for Treatment by Pulmonary Tuberculosis Patients: A Qualitative Study Based on the "Illness Behavior" Model

\author{
PEDRO HENRIQUE PEREZ DE MOURA ${ }^{1}$ \\ RILVA LOPES DE SOUSA-MUÑOZ ${ }^{2}$ \\ RACHEL MARTINS CANDEIA ${ }^{1}$
}

\section{RESUMO}

Objetivo: Avaliar como portadores de tuberculose pulmonar em tratamento narram suas experiências de doença, verificando-se, no seu discurso, as diferentes fases do "Comportamento de Enfermo", interpretadas na perspectiva da análise de conteúdo proposta por Bardin. Material e Métodos: Estudo qualitativo fundamentado na técnica da análise discursiva a partir de entrevistas com 16 pacientes com tuberculose pulmonar em tratamento ambulatorial no Hospital de Doenças Infecto-Contagiosas Clementino Fraga em João Pessoa, Paraíba. O referencial teórico foi o enfoque "Comportamento de Enfermo" proposto por Mechanic. Resultados: Observou-se busca tardia de atenção médica, procura inicial de hospitais gerais, com atraso no diagnóstico e limitação para o trabalho como fator determinante da aceitação do papel de doente e da necessidade de assistência médica. Ao receber o diagnóstico, observou-se temor de contágio de familiares e estigma em relação ao diagnóstico. As causas para a tuberculose foram atribuídas ao estilo de vida, condições climáticas, trabalho e problemas emocionais. $\mathrm{Na}$ fase de comportamento dependente, os pacientes referiram transferência do controle ao médico. Conclusão: Foram observadas diversas fases do "Comportamento de Enfermo", desde a negação dos sintomas até o ajustamento a esse papel. Após essa conformação, os pacientes manifestaram necessidade da legitimação de sua doença através do diagnóstico médico. Estes dados apontam para a necessidade de se ir além do quadro clínico para maior efetividade de tratamento.

\section{DESCRITORES}

Tuberculose. Papel do Doente. Pesquisa Qualitativa.

\begin{abstract}
Objective: To evaluate how pulmonary tuberculosis patients report their illness experiences as well as to verify the different phases of "illness behavior" in the perspective of content analysis as proposed by Bardin. Material and Methods: This was a qualitative study using the discourse analysis of interviews with 16 pulmonary tuberculosis patients undergoing Directly Observed Treatment Shortcourse (DOTS) at the Clementino Fraga Hospital of Infectious Diseases in João Pessoa, Paraiba, Brazil. This study was based on the theory of "illness behavior" proposed by Mechanic. Results: It was observed a late seeking for medical attention, initial demand for general hospitals, with a delay in diagnosis, and limitation to work as a determinant factor for the acceptance of the sick role and of the need for medical assistance. Upon receiving the diagnosis, there was fear of contagion to family and stigma regarding the diagnosis. The causes for tuberculosis were attributed to lifestyle, weather, work and emotional problems. As to the dependent behavior phase, patients reported transferring the control to the doctor. Conclusion: Diverse phases of the "illness behavior" were observed, since denial of symptoms until adjustment to this sick role. As such, patients reported the need for legitimizing their illness through the medical diagnosis. This study indicates the need for healthcare professionals to go beyond the clinical framework in order to reach a more effective treatment.
\end{abstract}

\section{DESCRIPTORS}

Tuberculosis. Sick Role. Qualitative Research.

1 Estudante de Medicina da Universidade Federal da Paraíba (UFPB), João Pessoa/PB, Brasil.

2 Professora Doutora do Departamento de Medicina Interna do Centro de Ciências Médicas da Universidade Federal da Paraíba (UFPB),João Pessoa/ PB, Brasil. 
$\mathrm{C}$ om o avanço da tecnologia médica que o método clínico proporcionou, além da fragmentação corpo-mente, houve a fragmentação do próprio corpo, pois a medicina tornou-se cada vez mais especializada ao longo do século XX. Porém, a despeito dos avanços no conhecimento biomédico, a abordagem semiológica e qualitativa de casos clínicos particulares continua sendo a base para identificação de problemas subjetivos que podem ter forte influência sobre a busca de tratamento e a adesão terapêutica (GROSSMAN, CARDOSO, 2006). No caso de uma doença infectocontagiosa crônica, como a tuberculose pulmonar, é fundamental conhecer a reação do paciente ao seu próprio quadro, para a consecução da integralidade no tratamento. Essa reação significa, no âmbito da linguagem, a construção de um sentido para a situação existencial do doente durante a quimioterapia antituberculose diretamente observada de seis meses de duração (JUNGES, BAGATINI, 2010).

A cura da tuberculose no Brasil ainda enfrenta um grande desafio: a falta de adesão dos pacientes ao tratamento. Este é o maior obstáculo para o controle e eliminação da doença (ASSUNÇÃO, SEABRA, FIGUEIREDO, 2009), estimando-se que a taxa de abandono do tratamento varia de 4,5 a 20,3\% nas diversas regiões do Brasil (VIEIRA, RIBEIRO, 2008; PAIXÃO, GONTIJO, 2007). Assim, a eficácia dos medicamentos não é suficiente para assegurar a cura da tuberculose, devendo ser valorizado o comportamento do doente no processo de cura, o qual constitui, provavelmente, o mais importante fator de sucesso terapêutico.

Atualmente uma das principais preocupações nos estudos sócio-antropológicos sobre saúde referese às pesquisas relacionadas à "experiência da doença". Nesse sentido, torna-se cada vez mais importante buscar abordagens qualitativas nas pesquisas sobre o problema, a partir das quais poderiam ser encontradas estratégias visando à conscientização do doente sobre essa enfermidade e seu tratamento. $\mathrm{O}$ estudo da narrativa do paciente diante do adoecimento e tratamento coloca sua experiência como elemento central, por valorizar a dimensão subjetiva do processo de adoecer e os seus aspectos relacionais e interpessoais (MAIA, STRUCHINER, 2010).

Para MECHANIC, (1995) essa narrativa da experiência do adoecimento pode ser interpretada através da perspectiva "Comportamento de Enfermo", que diz respeito ao processo pelo qual os sintomas são definidos, dotados de significação e socialmente rotulados, além de seguidos pela busca de assistência médica, através da qual o seu regime de vida é modificado.
O objetivo deste trabalho foi identificar as percepções de pacientes portadores de tuberculose pulmonar sobre sua doença e tratamento, verificandose, no seu discurso, as diferentes fases do "Comportamento de Enfermo".

\section{MATERIAL E MÉTODOS}

Trata-se de um estudo exploratório de natureza qualitativa, cujo projeto foi aprovado pelo Comitê de Ética em Pesquisa da Universidade Federal da Paraíba (Parecer número 187/2002).

Foram realizadas entrevistas individuais com pacientes portadores de tuberculose, com perguntas abertas orientadas pela "Escala de Avaliação do Comportamento de Enfermo do Indivíduo”.

Os 16 pacientes entrevistados apresentavam formas pulmonares da doença e cumpriam a segunda fase do tratamento ou do retratamento, sob regime de tratamento diretamente observado (DOT) no Hospital de Doenças Infecto-Contagiosas Dr. Clementino Fraga (HDICF). Foram excluídos aqueles pacientes com formas extra-pulmonares da doença e portadores do vírus HIV.

Os pacientes foram divididos nos grupos PT (primotratamento: esquema E-I) e RT (retratamento: esquema E-I-R ou esquema E-III). Os pacientes do grupo PT não realizaram tratamento anterior para tuberculose. No grupo RT, os pacientes já tinham recebido o esquema E-I por 30 dias ou mais, tendo abandonado, usado irregularmente a medicação ou apresentado falência terapêutica.

O referencial metodológico para análise do material obtido nas entrevistas foi a técnica da análise de conteúdo proposta por BARDIN, (1977). As entrevistas foram gravadas com o consentimento dos sujeitos e transcritas na íntegra. O referencial teórico adotado foi o enfoque "Comportamento de Enfermo" (Illness Behavior) de MECHANIC, (1995) que descreve as reações de um paciente à experiência de estar enfermo. Tais reações são formuladas em cinco etapas: (1) estágio de experiência dos sintomas; (2) estágio de acomodação ao papel de doente; (3) estágio de contato com a área médica; (4) estágio do papel de paciente-dependente; (5) estágio de recuperação.

Através da análise do conteúdo, que é um processo de redução de informação capaz de fazer uma análise qualitativa e quantitativa dos dados obtidos a partir da transcrição das entrevistas, as categorias temáticas que apareceram no texto foram classificadas com base na sua similaridade semântica, refletindo os padrões cognitivos do grupo estudado.

Para a escolha das unidades de análise e 
agregação a partir dos recortes do discurso de cada paciente entrevistado, as categorias foram agrupadas nos conjuntos de 1 a 5 com base no modelo teórico adotado.

Na coleta de dados em estudos qualitativos, a validade das informações está relacionada ao grau em que se captura o fenômeno em estudo. É possível conseguir esta validade, se a informação é obtida a partir dos próprios indivíduos que experimentam o fenômeno. Por esta razão, foi importante considerar a perspectiva do paciente sobre o processo de adoecimento e de busca de atenção.

O número de indivíduos estudado foi determinado de acordo com o critério de amostragem por saturação, usada para estabelecer o tamanho final de uma amostra em estudo com interrupção da captação de novos componentes (FONTANELLA, RICAS, TURATO, 2008).

Para garantir a validade das inferências, a organização de dados em categorias foi feita por consenso entre dois dos investigadores (C.E.F. e R.L.S.M.). Por sua vez, esta construção permitiu desenvolver uma tabela de duas entradas (paciente e categoria) para a análise quantitativa, para calcular as frequências das questões e fazer comparações (MOREIRA, 2002).

\section{RESULTADOS}

Foi realizada uma análise de conteúdo qualitativa (BARDIN, 1977) para se investigar os depoimentos dos
16 entrevistados. Com base nos discursos, emergiram cinco categorias temáticas e 15 subcategorias (Tabela $1)$.

Participaram deste estudo 16 pacientes, predominantemente do sexo masculino, idade entre 18 e 49 anos, até quatro anos de escolaridade e renda mensal de até um salário-mínimo (Tabela 2).

Os principais resultados da análise temática dos discursos estão apresentados conforme as cinco etapas do modelo "Comportamento de Enfermo" adotado:

\section{1- Percepção dos sintomas da doença}

Em relação à primeira fase do comportamento de enfermo do indivíduo - experiência dos sintomas -, na qual o indivíduo sente que "algo está errado consigo", observou-se que a tosse foi a manifestação mais frequente em ambos os grupos, ainda que não valorizada ( “...eu achava que era só uma tosse...”), e relatada por todos os pacientes como o sintoma mais persistente $\mathrm{e}$ repetitivo ( “...tossia todo dia, todo dia...” / “...eu já tinha dado umas 500 tossidas...”).

No grupo PT, a dor torácica foi o sintoma que mais incomodou ( "... mas o pior de tudo é a dor" / “... o meu pulmão começou a doer (...) era dor forte...”), seguida pela hemoptise. Foi observado também o sentimento de vergonha pela presença deste último sintoma ("... eu não digo que botei sangue porque tenho vergonha..." / “... estou escarrando, com licença da palavra, sangue...”). No grupo RT, figuraram a acentuada perda de peso e a hemoptise como os sintomas mais valorizados.

\begin{tabular}{|c|c|}
\hline Cateqorias & Subcategorias \\
\hline 1- Experiência de sintomas & $\begin{array}{l}\text { 1.1- Manifestaçóes } \\
\text { 1.2- Intensidadelfrequéncia }\end{array}$ \\
\hline 2- Acomodaçāo ao papel de doente & $\begin{array}{l}\text { 1.3-Reoercussoes } \\
\text { 2.1-Reconhecimento } \\
\text { 2.2. Resistência }\end{array}$ \\
\hline 3- Contato com o serviço de saúde & $\begin{array}{l}\text { 2.2- Resistencia } \\
\text { 3.1- Posto de Saùde } \\
\text { 3.2- Hospital } \\
\text { 3.3- Causa da tuberculose } \\
\text { 3.4- Discriminaçao sodal } \\
\text { 4.1- Médicolmedicacáa }\end{array}$ \\
\hline 4- Tratamento: papel de paciente dependente & $\begin{array}{l}\text { 4.2-Adesto } \\
\text { 4.3-Dificuldades } \\
\text { 4.4-Exoectativas }\end{array}$ \\
\hline 5- Recuparaçâo & $\begin{array}{l}\text { 5.1- Resultados } \\
\text { 5.2-Abandono }\end{array}$ \\
\hline
\end{tabular}




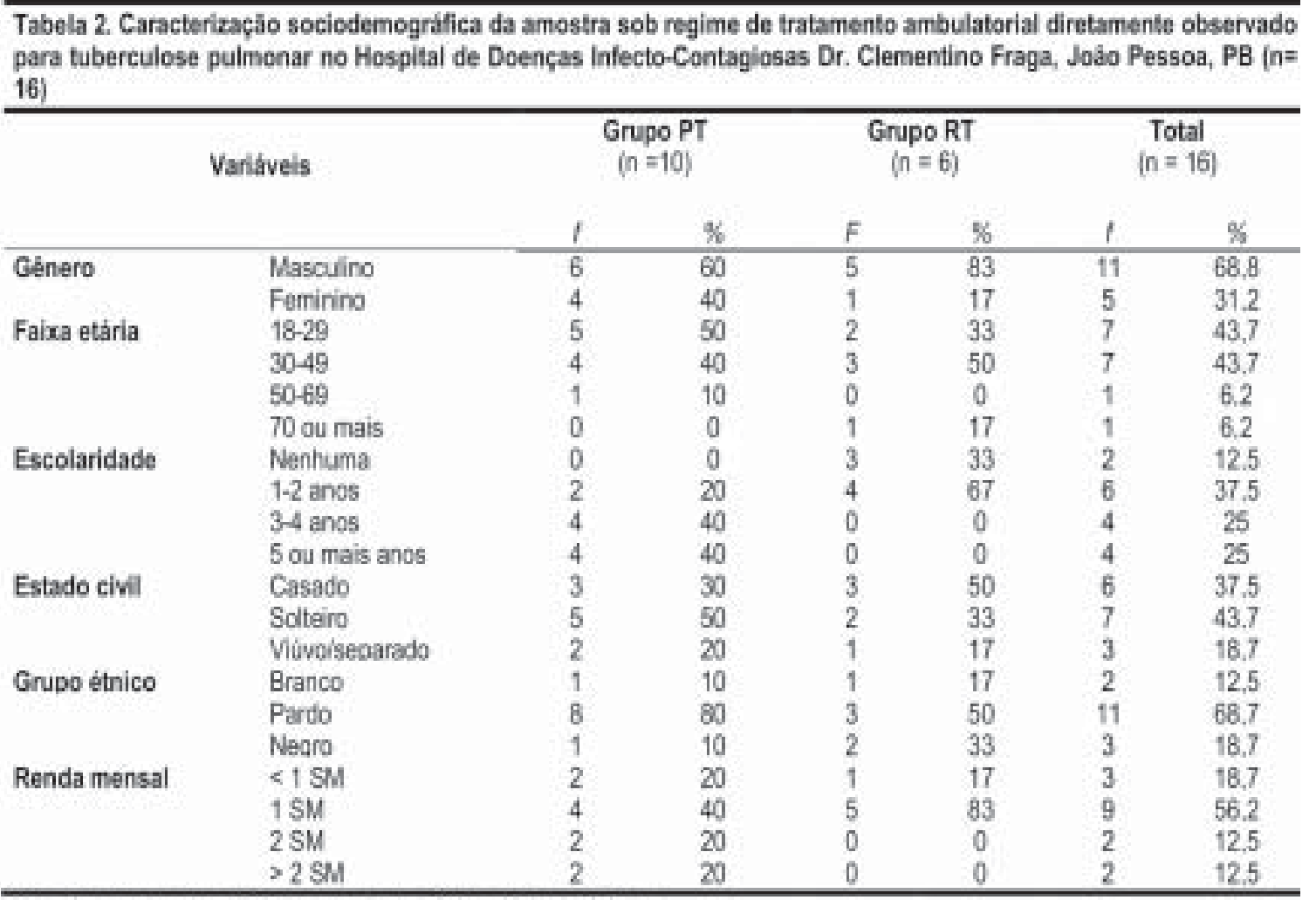

PT: primotratamento. RT: retratamento. SM: salarios-minimos.

Febre persistente e mal-estar geral também foram mencionados pela maior parte dos doentes dos dois grupos, porém a "materialização" da enfermidade parece ter-se revelado a partir do momento em que apresentaram hemoptise, dor torácica ou notável perda de peso com limitação para o trabalho ( "... tinha vez que não dava nem p'rá me levantar (...) tem muita coisa que a gente não pode fazer..." / "...passei dois meses sem trabalhar...").

Essas últimas manifestações foram as que desencadearam a busca de atenção médica pela maioria dos pacientes, ainda que esta tenha sido tardia em relação ao início do quadro clínico (tosse e febre). Os pacientes apresentaram tosse por semanas a vários meses, sem se preocupar em buscar um serviço de saúde (“... a tosse já tem um tempão...” / “... tosse velha, catarro no peito..." / “... já fazia um bocado de tempo que eu já tinha essa tosse...”).

\section{2- Acomodação ao papel de doente}

A partir de um determinado momento, os indivíduos passaram a reconhecer que estavam doentes e que precisavam de auxílio médico, passando a "acomodar-se" ou "amoldar-se" a esse papel ( "... eu disse a ela 'agora eu vou [ao médico], agora é sério mesmo'..." / “... eu disse 'eu estou mal'...” / “... cada vez mais foi se agravando, agravando (...) quando eu não aguentei mais, resolvi procurar o médico” / “... o jeito foi procurar o médico...").

Nessa segunda fase do comportamento de enfermo, a maioria dos pacientes recorreu a uma combinação de alternativas antes de receber o diagnóstico, incluindo o uso de chás e "lambedor" ( "... eu tomava um chá...”, “... esse lambedor que a pessoa faz...”) antes de buscar um serviço de saúde. Nesse sentido, observou-se um conjunto de relatos referentes à negação dos sintomas da doença e ao seu diagnóstico ( "...eu pensava que era uma gripezinha qualquer, uma tosse..." / “...não sabia a gravidade dela...” / “...pensava que era outra coisa, um 'negociozinho'...” / “...não achei que ia ter uma doença dessa...”).

\section{3- Contato com o serviço de saúde}

Durante a terceira fase, na qual se deu a procura de auxílio médico, observou-se que os pacientes buscaram inicialmente hospitais gerais da cidade, sendo internados em tais serviços. Apenas um dos pacientes chegou ao HDICF encaminhado a partir de um posto de saúde.

O diagnóstico de tuberculose passou despercebido na maioria das avaliações médicas iniciais, sendo confundido com outras doenças respiratórias (sinusite, 
pneumonia, asma) e até com dengue, para as quais os pacientes receberam medicamentos. Sem melhora, foram, então, encaminhados ao HDICF, ocasião em que necessitavam de internamento pela gravidade clínica apresentada. Este aspecto foi mencionado por 12 dos pacientes, e pode ser ilustrado pela afirmação de um dos entrevistados: “... eu estava me tratando no médico errado (...) eu já tinha corrido dois hospitais e não tinha resolvido...".

Além disso, alguns pacientes tenderam a ocultar as manifestações de tuberculose por temerem o rechaço de seus familiares e amigos pelo estigma da doença (“... muitos amigos meus quando souberam que eu estava com essa doença, para eles eu tinha morrido..." / “... nenhum [familiar] não vai nem lá em casa...” / “... ficavam me chamando já era de tuberculoso...” / “...eu não demonstro porque fico mais dentro de casa (...) eu não quero demonstrar a mais ninguém a minha doença...”). Muitos deles apresentaram temor de contagiar seus familiares, o que ficou evidenciado principalmente pela separação dos fômites ( “... o meu copo, minha colher de casa são separados, já para não prejudicar ninguém...”), afastamento (“... eu digo que não pode ficar muito perto de mim não..."), ou uso de manobras para evitar a transmissão ("...a primeira coisa que eu fiz foi pegar uma toalha e andar com ela assim no ombro, aí eu dobrava assim e quando os meninos vinham falar comigo, eu pegava e colocava no rosto...").

A partir do momento em que passaram a conhecer o diagnóstico de tuberculose, os pacientes perceberam a doença como um mal que levava ao consumo orgânico ( "... eu fiquei que nem um fósforo..." / “... [a doença] vai secando o cara (...) seca, seca, seca, quando vai correr está da grossura de uma caneta...” / “... se eu ficava só em casa chegava um momento que eu ficava pele e osso..."), de natureza contagiosa, como já foi mencionado, e que assusta tanto a eles mesmos como a sociedade por sua transmissibilidade e gravidade ("... ela é grave, quando ela contagia (...) se não tiver noção do que ela causa, você morre" / "... esta doença, se a pessoa não cuidar, morre...)". Contudo, ainda que soubessem da natureza contagiosa da doença, para eles as causas primárias desta eram o tabagismo, o alcoolismo, a alimentação deficiente, as condições climáticas adversas, o tipo de trabalho, os problemas emocionais, o estilo de vida, etc. Apenas um entrevistado relatou conhecer sua etiologia microbiana.

\section{4- Comportamento de paciente dependente}

Nesta quarta fase, os pacientes apresentaram relatos referentes à transferência do controle ao médico, demonstrando aceitação do tratamento prescrito. Reportaram a ideia de que o médico detinha o conhecimento necessário para determinar o tratamento e mencionaram necessidade de obediência ao esquema terapêutico ( "... estando com os médicos, me sinto seguro..." / "... estou com as pessoas competentes que o Senhor preparou..." / "... eu venho tomar o remédio porque ela [a médica] disse que eu tenho que vir..."). Durante o tratamento específico nesta fase, nenhum paciente se referiu aos medicamentos por seu nome, citando apenas suas características, como cor, quantidade ou apresentação ("...são três [comprimidos] do azul e dois do vermelho..." / “...vou ter que tomar 90 injeções e 10 comprimidos todo dia...”).

Notaram-se diferenças entre os pacientes dos grupos PT e RT em relação à melhora clínica subjetiva alcançada nos primeiros três meses de tratamento. Todos os pacientes PT relataram completa melhora clínica ("...mil por cento eu estou bem...” / “... não sinto mais nada não...” / “...não estou mais sentindo que tenha [a doença]...” / “...parece até que eu nem tive isso...”), enquanto os pacientes RT não expressaram de igual maneira a melhora sentida, relatando uma melhoria parcial e relativa (“...melhorou um pouco...” / “...não me recuperei, a doença só afastou...” / “...graças a Deus, p'rá vista do que eu 'tava, eu 'tou mais melhorada...").

As dificuldades referidas no enfrentamento da terapêutica foram de natureza operacional ou administrativa, sendo pouco mencionados os efeitos colaterais dos medicamentos. Mesmo com os subsídios para transporte recebidos da Secretaria de Saúde, os entrevistados expressaram dificuldades referentes ao deslocamento diário de sua casa até o HDICF ( “... é meio cansativo, o ônibus de... é muito demorado" / (...) só faltei um dia porque eu perdi o vale [valetransporte]...”), bem como a interferência nas suas atividades laborativas ( "... estou sem trabalhar por conta do tratamento (...) tem que vir para aqui tomar o remédio..." / “... me dificulta trabalhar, todo dia saio daqui quase nove horas, dez"), omissão das doses durante os finais de semana e feriados ( “...não trouxe remédio de lá, fiquei três dias em casa sem tomar o remédio"), a posologia ("...agora isso [tomar remédios], é todo dia..." / “... não é brincadeira, tomar 10 comprimidos todo dia em jejum... / “...é remédio demais!”), a duração do tratamento ( “...o que está me preocupando é assim, se esse tratamento vai demorar muito..."), eventual falha na entrega dos remédios ( “... ela [a enfermeira] não entregou o remédio") e a dificuldade de acesso ao HDICF ( “...eu sei o sofrimento para eu conseguir [me tratar no HDICF], mesmo estando eu assim doente...” / “...toda vez que eu vinha p'ro hospital [Clementino Fraga] tinha dez, vinte, na minha frente (...) eu disse 'ah, vou embora, não tenho paciência, vou embora'...”). Apareceram dois relatos referentes aos efeitos colaterais ("... [a medicação] $m e$ dá um mal estar medonho...” / “...é muito forte “).

No grupo PT, a maioria dos pacientes referiu que a tuberculose seria curada se houvesse o seguimento da recomendação médica, ressaltando a necessidade de se concluir o tratamento. No grupo RT, observou-se que a confiança na cura foi menor, havendo relatos de 
que a doença talvez nunca fosse curada ( “... eu vou voltar p'ro hospital, vou ficar doente de novo" / "... vamos esperar p'rá ver no que é que vai dar...). No grupo RT, diversamente do grupo PT, foi frequente a falta de adesão quanto a medidas de ordem nãofarmacológica, como o abandono do tabagismo e alcoolismo.

\section{5- Abandono do papel de doente}

No momento das entrevistas todos os pacientes ainda recebiam atenção médica e se encontravam aproximadamente na metade do curso terapêutico (grupo PT) e na sua terça parte (grupo RT), portanto ainda não haviam recebido alta, sendo considerados pelo sistema de saúde como "doentes".

As expressões dos pacientes que refletem a melhora subjetiva foram mencionadas anteriormente e representam a melhora sintomática que ocorre na primeira fase da quimioterapia, porém não se observaram unidades temáticas relacionadas com a intenção de abandonar o papel de doente no grupo PT. No grupo $\mathrm{RT}$, foram encontradas poucas expressões indicativas de tendência ao abandono do papel de doente ("... eu disse: 'doutora, a senhora já não cortou a medicação? Eu não vou tomar mais não'...” / “[o tratamento] ...já era p'rá ter acabado há muito tempo).

\section{DISCUSSÃO}

No conjunto de narrativas analisadas, foram identificadas as seguintes unidades temáticas, que se sucederam na cronologia das falas dos entrevistados: (1) negação dos sintomas, (2) tendência a ocultar as manifestações de tuberculose; (3) busca tardia de atenção médica; (4) limitação para o trabalho como fator determinante da aceitação do papel de doente; (5) procura inicial de hospitais gerais da cidade com atraso no diagnóstico; (6) papel de paciente-dependente com transferência do controle ao médico; (7) percepção da tuberculose como uma doença mortal se não tratada; (8) interferência no trabalho pela execução do DOT; e (9) constrangimento pela posologia e duração do tratamento.

Os pacientes entrevistados apresentaram expressões compatíveis com o modelo teórico adotado, de "Comportamento de Enfermo", além de declarações características da narrativa da experiência de doente de tuberculose. Esses modos de encarar o processo de adoecimento pela tuberculose podem ter apresentado grande influência sobre a busca de atenção médica para obter tratamento, pois a percepção do estigma social relacionado ao diagnóstico e a falta de conscientização podem contribuir para o diagnóstico tardio e baixa taxa de detecção de casos de tuberculose (ASCUNTAR et al., 2010; RUFFINO-NETTO, 2002).

A experiência de cada paciente entrevistado sobre a sua doença foi rica em detalhes sobre o início aos primeiros sintomas, sua evolução, até a fase de incapacidade, passando pelas mudanças vividas durante o adoecimento. Percebeu-se como este provocou nos indivíduos uma nova identidade, chamada de "pessoa doente" e, a partir daí, levaram-nos a representar o seu "Comportamento de Enfermo".

A tosse foi o sintoma mais precoce e frequente entre os pacientes entrevistados, porém foi considerado por todos como um sintoma banal. Por outro lado, a dor, que é uma das manifestações mais comuns na prática clínica, por sua própria natureza, não foi tolerada durante muito tempo sem que se buscasse auxílio médico. Além disso, parece fazer parte do universo de representações sobre as doenças de modo geral, a ideia de que estas se evidenciam através de dor.

Para os entrevistados, a tuberculose sugeriu inicialmente a possibilidade de uma "gripe", determinando uma atitude de banalização em relação à sintomatologia, que resultou apenas em tentativas de tratamento alternativo no âmbito doméstico. A hemoptise, entretanto, sintoma bastante associado ao diagnóstico de tuberculose pelo leigo, alarmou por indicar a presença de gravidade, sendo reconhecido como um dos sintomas desencadeadores da busca de atenção médica.

Contudo, sabe-se que diferentes pessoas percebem e avaliam os sintomas e reagem à doença de maneiras diversas. Nesse sentido, o conhecimento e as crenças que os pacientes tiveram sobre a doença e seu tratamento foram fatores importantes para a aceitação final do papel de doente. Nesse sentido, a importância que as pessoas conferem à sua saúde e aos cuidados para preservá-la, depende, em especial, de sua situação socioeconômica, além dos conhecimentos do senso comum, de hábitos e de atitudes e crenças que são aprendidos culturalmente (MECHANIC, 1995). É dessa forma que em geral passam a ser incorporada à compreensão do "sentir-se doente", modalidades que se situam além da definição de que o indivíduo "tem uma doença", mas que dependem dos significados que são atribuídos ao processo de adoecer em uma cultura determinada.

RAMOS, (2007) salienta a importância dos três diferentes conceitos atribuídos à doença (KLEINMAN, 1978; KLEINMAN, 1980), que incluem "disease", "illness" e "sickness". São três realidades diferentes que interagem entre si: a realidade biofísica, psicológica e social. Estas realidades expressam-se através de dimensões diferentes, ou seja, "ter uma doença", "sentirse doente" e "comportar-se como doente". "Disease" representaria, na dimensão biomédica, a realidade biológica de uma alteração objetivamente verificável no organismo, significa "a doença do médico" - esta concepção é a que ocorre quando o doente chega ao serviço de saúde; por outro lado, "illness" representaria a realidade psicológica, a dimensão subjetiva, traduzindo as experiências individuais do doente em consequência 
das alterações percebidas por ele e das interações com o seu meio, significa a "doença do doente" - esta seria a concepção que levaria ou não o doente ao serviço de saúde; contudo ainda há o "sickness", que seria a realidade ou processo psicossocial, representando uma identidade social, um estatuto ou um papel, o "comportar-se como doente", que atravessa todo o processo.

Por outro lado, depois que se deu a conformação ao papel de doente, os pacientes buscaram confirmação, ajuda, garantias e, em última instância, o que MECHANIC, (1995) define como "validação provisional", ou seja, uma forma de "dispensa" temporária de sua obrigação das atividades normais, pois a saúde pode, em última instância, ser definida como "o estado de capacidade ótima de um indivíduo para o desempenho eficiente dos papéis e das tarefas para os quais foi socializado" (MECHANIC, 1995).

Esse foi um dos aspectos também observados no processo de busca de assistência médica no presente estudo, quando os indivíduos passaram a se sentir e a se auto referir como "doentes", o que correspondeu ao momento em que a enfermidade repercutiu fortemente sobre a sua capacidade física, comprometendo significativamente a dimensão laborativa de sua vida. Isso evidencia que, no grupo entrevistado, cujo nível socioeconômico era desfavorável, o referencial para a saúde parece ser representado pela capacidade de poder desenvolver as atividades para a manutenção do trabalho.

Pareceu não existir um limite claro entre o "estar doente" e o "estar são". Nesse contexto, "estar são" pode significar "sentir-se bem" - o que é uma noção subjetiva e não passível de mensuração -, pode significar não apresentar sintomas e sinais de doença, mas pode também, em última instância, significar que o sujeito consegue desempenhar o seu trabalho (ALVAREZGORDILLO et al., 2000). Como tal incapacidade não pôde ser superada pelos enfermos, eles necessitaram da "legitimação" de sua enfermidade pela sociedade, através de um diagnóstico médico e, sobretudo, de um processo terapêutico para sua recuperação (ALMEIDAFILHO, MONTENEGRO, SANTOS, 2009).

Assim, o estado de "sentir-se doente" relacionou-se aos significados que o paciente, assim como sua família e comunidade, atribui ao processo de enfermar. Tal papel assumido pelo doente, denominado "Comportamento de Enfermo" é, em última instância, o papel que a sociedade atribui à pessoa pelo fato de estar enferma. Nesse sentido, em contraposição à visão positivista da doença, percebe-se que os termos "enfermar" e "sentir-se enfermo" têm significações diferentes (MECHANIC, 1995).

No presente estudo, observou-se que a maioria dos pacientes procurou assistência hospitalar inicial, e não um serviço de atenção primária ou básica, "porta de entrada" no Sistema Único de Saúde. Esse fato pode ser explicado, em parte, pela gravidade do quadro clínico, pois quase todos os pacientes procuraram atenção médica quando estavam intensamente sintomáticos e em estado geral comprometido pela doença. É importante enfatizar a importância desse aspecto no controle da tuberculose, pois a procura precoce de assistência à saúde em relação ao início dos sintomas, que nesse caso poderia ser a ocorrência de tosse e febre com duração maior que 15 dias sem outra causa aparente, reduziria o risco de morte e de disseminação da doença.

Nesse sentido, CABRAL et al., (2011) salientam que a preocupação sobre como e em que momento as pessoas procuram ajuda para resolver suas demandas ou problemas de saúde tem estado cada vez mais presente em estudos sobre planejamento, organização e avaliação de serviços assistenciais de saúde. A intenção é subsidiar a escolha de estratégias adequadas que garantam acesso aos usuários em momento oportuno, propiciando vínculo com a equipe de profissionais de saúde e, consequentemente, adesão ao tratamento proposto.

O conteúdo das frases temáticas revela um movimento que impulsiona a pessoa a buscar ajuda no campo da saúde, e que este não é um processo fácil de ser levado adiante quando se trata de tuberculose. Como os parâmetros para a definição dessas situações-limite são diferentes para cada indivíduo, e como dependem de sua posição na sociedade, é fundamental entender que, muitas vezes, a demora para a procura na assistência não se deve apenas a uma questão de "negligência", mas a vários outros fatores que envolvem o cotidiano dos doentes (CAMPINAS, ALMEIDA, 2004).

A literatura socioantropológica utiliza o termo "itinerário terapêutico" para definir este percurso. Itinerários terapêuticos são constituídos por todos os movimentos desencadeados por indivíduos ou grupos na preservação ou recuperação da saúde, que podem mobilizar diferentes recursos, que incluem desde os cuidados caseiros e práticas religiosas, até os dispositivos biomédicos predominantes (atenção primária, urgência etc). Referem-se a uma sucessão de acontecimentos e tomada de decisões que, tendo como objeto o tratamento da enfermidade, constrói uma determinada trajetória (CABRAL et al., 2011).

Nesse sentido, BERTOLOZZI, (1998) afirma que o indivíduo que tem uma necessidade de saúde geralmente se apresenta como vulnerável e entregue aos serviços de saúde. Essa autora define o "paciente" como expropriado de sua condição de cidadão e que suas crenças são desvalorizadas ou anuladas, não havendo consideração de seus sentimentos e carências nos serviços de saúde. Assim é que geralmente se verifica neste âmbito a definição de intervenções segundo a ótica exclusivamente técnica, sem a participação ativa do doente no processo terapêutico.

Durante a fase de paciente dependente, percebeu-se que foi importante a relação entre médico (a) e paciente, já que esta interação pode influir na comunicação e, consequentemente, na adesão 
terapêutica. MECHANIC, (1995) parte da hipótese de que, em muitos casos, devido a diferenças de ponto de vista quanto ao significado da enfermidade e aos distintos conceitos relacionados com a atenção médica, surgem conflitos entre o médico e o paciente que podem interferir no tratamento.

Somente quando chega a esta etapa, o doente se converte em um "paciente". No entanto, o fato de que uma pessoa entra em contato com um médico não significa necessariamente que esteja disposta a aceitar as suas recomendações e a renunciar a algumas de suas prerrogativas, adotando um papel de "dependência" em relação à atenção médica. $\mathrm{O}$ futuro paciente geralmente assume este papel de dependente com certa ambivalência. Contudo, mesmo que deseje evitar este papel, é provável que posteriormente o considere como a única maneira de recuperar suas funções normais e voltar a usufruir sua plena saúde (GONÇALVES, 2000).

Conforme PAIKIN, LEONOR, (1985) as características sociodemográficas e sociais dos indivíduos também determinam a maior ou menor dependência por parte do paciente. Os sujeitos de nível socioeconômico baixo e os idosos reagiriam mais frequentemente de acordo com a posição de dependência que os indivíduos de nível socioeconômico médio e os mais jovens. Também são fatores importantes durante esta etapa aqueles que afetam o ajuste do indivíduo a seu papel de enfermo, como foi relatado por alguns dos doentes entrevistados, tais como as barreiras físicas, administrativas, psicológicas e sociais que pareceram interferir no curso do tratamento.

Os pacientes entrevistados revelaram representações semelhantes no que se refere à mescla de conteúdos arcaicos e de conteúdos atuais sobre conhecimentos a respeito da tuberculose. Observou-se que a doença foi relacionada principalmente à perda de peso, à hemoptise, ao medo do contágio/transmissão, à noção de que essa enfermidade produz um estado de "consumo orgânico", a diversos constrangimentos e ao estigma social, assim como à possibilidade de a doença terminar por conduzir à morte (SOARES, 1994).

Por outro lado, os doentes do grupo PT disseram acreditar que a cura seria produzida pelo tratamento medicamentoso que estava em curso, o que foi menos observado no grupo RT. Não se detectaram expressões indicativas de tendência ao abandono terapêutico no grupo PT, embora alguns pacientes do grupo RT, provavelmente pela experiência anterior mal sucedida ou por possuírem problemas psicossociais mais importantes - o que provavelmente influenciou no abandono ou irregularidade da primeira tentativa de tratamento -, tenham se mostrado mais pessimistas quanto à obtenção da cura.

Destaca-se ainda que o estigma em relação ao diagnóstico da tuberculose não se revelou somente da perspectiva da coletividade para com o doente, mas a relação inversa também pareceu ocorrer, em conformidade com o que observaram ASSUNÇÃO, SEABRA,
FIGUEIREDO, (2009). Para BERTOLOZZI, (1998) o preconceito que emerge do próprio doente evidentemente tem raiz diferente daquele que se configura por parte da coletividade em relação a ele. Os próprios doentes passaram a assumir atitudes de afastamento, possivelmente para evitar entrar em confronto com uma questão que para eles mesmos é difícil de ser incorporada no cotidiano.

Parece não haver dúvida de que é muito difícil localizar as situações ou condições que levariam os pacientes a deixarem de completar o seu tratamento para tuberculose. PERINI, (1998) abordou o problema do abandono do tratamento da tuberculose em Belo Horizonte - MG, com base na Teoria das Representações Sociais sobre a doença, verificando que a desinformação e a auto avaliação da eficácia pelo próprio paciente foi o fator condicionante fundamental para o abandono da terapêutica. $\mathrm{O}$ citado autor considera que sentimentos de medo e vergonha constituem fatores agravantes para as dificuldades de retorno ao serviço de saúde, revelando problemas na relação médico-paciente e barreiras culturais para percepção dos direitos pelo próprio usuário no sistema de saúde.

ODOT tem representado uma maneira de garantir a adesão do paciente ao tratamento medicamentoso. Contudo, o termo "adesão" procura ressaltar a perspectiva do paciente, como um ser capaz de uma decisão consciente e responsável por seu tratamento (BERTOLOZZI, 1998). Assim, o paciente não é visto apenas como um cumpridor passivo de uma prescrição medicamentosa. Além disso, o conceito de "adesão" incorpora uma dimensão do comportamento pessoal não apenas no que se refere à tomada da medicação, mas também no que se relaciona ao seguimento de dietas, abandono de hábitos, como tabagismo ou alcoolismo, e mudanças de estilo de vida, segundo o aconselhamento de profissionais de saúde.

No caso de pacientes em tratamento para tuberculose, o "papel de doente" é prolongado (no mínimo, de seis meses) e, neste sentido, outros fatores podem determinar sua maior ou menor aceitação do tratamento, como alcoolismo, drogadição, história prévia de baixa adesão (pacientes em RT da presente amostra), distúrbio emocional coexistente, falta de transporte para dirigir-se à unidade de saúde, insatisfação com a assistência do serviço de saúde, carência de suporte familiar, desemprego e baixa renda (PAIXÃO, GONTIJO, 2007; ABEBE et al., 2010). O Ministério da Saúde (BRASIL, 2011) destaca como fatores relacionados à não adesão, a concepção popular de saúde e de doença, a relação médico-paciente, internação prévia, melhora clínica após o início do tratamento (concepção de doença ligada à presença de sintomas), rejeição ao serviço de saúde e problemas de subsistência, como a falta de alimentos.

A partir do modelo teórico descrito por MECHANIC, (1995) percebe-se que a questão primordial que se coloca é fazer com que a pessoa se sinta suficien- 
temente estimulada para desenvolver um "comportamento" apropriado, ou seja, que o doente se torne consciente e responsável sobre a recuperação de sua própria saúde. Nesse sentido, do conjunto de depoimentos analisados no grupo PT, observou-se que fatores que pareceram demarcar fortemente alguns comportamentos de doente durante a sua terapêutica foram as suas próprias expectativas em relação ao tratamento e à cura, assim como à motivação para recuperação da saúde e as vantagens pessoais de seguir com a terapêutica até o final.

Talvez em virtude dessa expectativa positiva em relação à terapêutica da tuberculose, os efeitos colaterais tenham sido pouco expressos. Sabe-se que os medicamentos que apresentam muitas reações ou efeitos colaterais, como é o caso dos tuberculostáticos, repercutem definitivamente no potencial da adesão ao tratamento. Porém, a relação dos pacientes com os medicamentos nem sempre é simples e não se restringe ao seu consumo e às reações advindas desse consumo. Talvez por necessitarem de um convívio mais prolongado com a equipe de saúde (estratégia DOT), alguns pacientes tenham evitado criticá-la, ou reclamar do tratamento, suportando-o sem queixas.

Entre as estratégias para incrementar a adesão ao tratamento da tuberculose no regime DOT, os pacientes incluídos nesta amostra contavam com uma combinação delas, tais como a supervisão diária da dose, a ajuda de custo para o transporte e educação com palestras e orientações por parte da equipe de saúde. Contudo, além do trabalho educativo contínuo junto ao doente, é importante partir também de um levantamento das expectativas trazidas pelo mesmo para, então, reconstruir novas relações entre tratamento-sintoma e saúde-sintoma sobre tais expectativas (ALMEIDAFILHO, MONTENEGRO, SANTOS, 2009; GONÇALVES, 2000).

Na medida em que essas necessidades não foram expressas efetivamente pelo doente, ou foram expressas, mas não decodificadas como necessidades pela equipe de saúde, podem ter passado ao largo do processo terapêutico, repercutindo na "aceitação" da doença. Facilitar essa elaboração é tarefa dos trabalhadores de saúde, que podem buscar entender o significado da doença para a pessoa, não reduzindo a enfermidade a sinais e sintomas (PIOVESAN, TEMPORINI, 1995).

A compreensão sobre como as pessoas e os grupos sociais realizam escolhas e aderem ou não aos tratamentos, ou seja, como constroem seus itinerários terapêuticos, é fundamental para orientar as novas práticas em saúde. No geral, as dimensões relativas aos contextos de vida dos usuários e suas histórias escapam aos serviços e aos profissionais de saúde, embora sejam elas que definem as possibilidades de oferta e acesso aos serviços de saúde (MANGIA, MURAMOTO, 2008). Não obstante o desejo de gestores e profissionais de serviços de saúde, os caminhos percorridos por pessoas em busca de cuidados terapêuticos não necessariamente coincidem com esquemas ou fluxos pré-determinados. Suas escolhas expressam construções subjetivas individuais, e também coletivas, acerca do processo de adoecimento e de formas de tratamento (CABRAL et al., 2011).

Pelo próprio fato de ser a tuberculose uma doença de cunho eminentemente social, há que buscar compreender o que ocorre com o paciente nas diversas fases do "Comportamento de Enfermo" vivenciadas por ele, desvelando as suas necessidades que, como pode ser visto, transcenderam as fronteiras que tradicionalmente a clínica tem colocado, mas que se referiram à noção de contágio, de afastamento, de isolamento e de discriminação, uma vez que esses conceitos são verbalizados pelos entrevistados, mas que muitas vezes não são abordados ou valorizados pelos profissionais de saúde.

Assim, a assistência e o tratamento do paciente portador de uma doença de longa duração, sobretudo uma enfermidade como a tuberculose, ainda cercada de estigmas sociais apesar da existência de cura atualmente, deve favorecer a adaptação a essa condição, instrumentalizando-o para desenvolver mecanismos que permitam ênfase na integridade do ser e as representações do seu mundo, em contraposição à condição reduzida de portador de sinais, sintomas e doenças.

\section{CONCLUSÕES}

Nas narrativas do grupo estudado, foram observadas diversas fases do "Comportamento de Enfermo", desde a negação dos sintomas até a fase de ajustamento a esse papel. Após essa conformação, os pacientes manifestaram necessidade da legitimação de sua doença através do diagnóstico médico. Estes dados proporcionam uma reflexão e análise sobre a perspectiva do paciente diante da tuberculose pulmonar.

A contribuição mais significativa deste estudo foi desvelar a importância do "Comportamento de Enfermo" através do relato das suas experiências sobre as dificuldades vivenciadas durante a evolução da doença, levando a uma tardia procura de assistência. $\mathrm{O}$ presente trabalho, em consonância com os achados da literatura, aponta para a necessidade de rever as práticas adotadas nos serviços de saúde, restritas ao próprio quadro clínico.

Mais intervenções qualitativas centradas no paciente e na atenção às barreiras estruturais são necessárias para melhorar a adesão e diminuir a carga global da doença. É necessário que médicos e demais profissionais de saúde aprofundem seus conhecimentos a respeito dos fenômenos sociais que envolvem a problemática da tuberculose pulmonar e como os pacientes vivenciam sua experiência e como atribuem significações a esta. 


\section{REFERÊNCIAS}

1- ABEBE G, DERIBEW A, APERS L, WOLDEMICHAEL K, SHIFFA J, TESFAYE M, et al. Knowledge, health seeking behavior and perceived stigma towards tuberculosis among tuberculosis suspects in a rural community in southwest Ethiopia. PloS One, 5(10): e13339, 2010.

2- ALMEIDA-FILHOAJ, MONTENEGRO HRA, SANTOSTCF. A nova ordem no combate à tuberculose no Brasil: implicações para a enfermagem. Rev. Rene, Fortaleza, 10(1): 114-123, 2009

3- ALVAREZ-GORDILLO GC, ALVAREZ-GORDILLO JF, DORANTES-JIMÉNEZ JE, HALPERIN-FRISCH D. Percepciones y prácticas relacionadas con la tuberculosis y la adherencia al tratamiento en Chiapas, México. Salud pública Méx., 42(6): 520-528, 2000.

4- ASCUNTAR JM, GAVIRIA MB, URIBE L, OCHOA J. Fear, infection and compassion: social representations of tuberculosis in Medellin, Colombia. Int. J. Tuberc. Lung Dis, 14(10): 1323-1329, 2010.

5- $\quad$ ASSUNÇÃO CG, SEABRA JDR, FIGUEIREDO RM. Percepção do paciente com tuberculose sobre a internação em hospital especializado. Ciencia y Enfermeria, 15(2): 69-77, 2009.

6- BARDIN L. Análise de Conteúdo. Lisboa: Edições 70, 1977. p. 169-184.

7- BERTOLOZZI MR. A Adesão ao Programa de Controle da Tuberculose no Distrito Sanitário do Butantã. [Tese de Doutorado]. São Paulo: Universidade de São Paulo; 1998. 246p.

8- BRASIL. Ministério da Saúde. Secretaria de Atenção à Saúde. Departamento de Atenção Básica. Tuberculose na Atenção Primária à Saúde / Ministério da Saúde, Secretaria de Atenção à Saúde, Departamento de Atenção Básica. 2a. ed. Brasília: Ministério da Saúde, 2011.

9- CABRAL ALLV, MARTINEZ-HEMAEZ A, ANDRADE EIG, CHERCHIGLIA ML. Itinerários terapêuticos: o estado da arte da produção científica no Brasil. Ciênc. saúde coletiva, 16(11): 4433-4442, 2011.

10- CAMPINAS LLSL, ALMEIDA, MMMB. Agentes Comunitários de Saúde e o acolhimento aos doentes com tuberculose no Programa Saúde da Família. Bol Pneumol Sanit, 12(3): 145-154, 2004.

11- FONTANELLABJB, RICAS J, TURATO ER. Amostragem por saturação em pesquisas qualitativas em saúde: contribuições teóricas. Cad. Saúde Pública, 24(1): 1727, 2008.

12- GONÇALVES HA tuberculose ao longo dos tempos. Hist. cienc. saude-Manguinhos, 7(2): 305-327, 2000.

13- GROSSMAN E, CARDOSO MHCA. As narrativas em medicina: contribuições a prática clínica e ao ensino médico. Rev. Bras. Educ. Med., 30 (1): 6-14, 2006.

14- JUNGES JR, BAGATINI T. Construção de sentido nas narrativas de doentes crónicos. Rev. Assoc. Med. Bras., 56(2): 179-85, 2010.

15- KLEINMAN A. Culture and healing in Asian societies: anthropological, psychiatric, and public health studies. Boston: G. K. Hall, 1978, 462p.
16- KLEINMAN A. Patients and healers in the context of culture: an exploration of the bordeland between anthropology, medicine and psychiatry. Califórnia: University of California Press, 1980, 427p.

17- MAIA F, STRUCHINER M. Utilização dos weblogs e de comunidades do orkut como ferramentas pedagógicas em cursos da área da saúde. Interface (Botucatu), 14 (35): 905-18, 2010.

18- MANGIA EF, MURAMOTO MT. Itinerários terapêuticos e construção de projetos terapêuticos cuidadores. Rev. Ter. Ocup. Univ., 19(3): 176-182, 2008.

19- MECHANIC D. Sociological dimensions of illness behavior. Soc. Sci. Med., 41(9): 1207-16, 1995.

20- MOREIRA DA. O método fenomenológico na pesquisa. São Paulo: Pioneira Thomson, 2002, 152p.

21- PAIKIN S, LEONOR M. Expectativas del rol del enfermo. Acta psiquiátr. psicol. Am. Lat., 31(4): 291-7, 1985.

22- PAIXÃO LMN, GONTIJO ED. Perfil de casos de tuberculose notificados e fatores associados ao abandono, Belo Horizonte, MG. Rev. Saúde Pública, 41(2): 205-13, 2007.

23- PERINI E. O abandono do tratamento da tuberculose: transgredindo regras, banalizando conceitos. [Tese de Doutorado]. Belo Horizonte: Escola de Veterinária, UFMG; 1998. 218p.

24- PIOVESAN A, TEMPORINI ER. Pesquisa exploratória: procedimento metodológico para o estudo de fatores humanos no campo da saúde pública. Rev. Saúde Pública, 29(4): 318-325, 1995.

25- RAMOS N. Comunicação e interculturalidade nos cuidados de saúde. Psycologica, 45: 147-69, 2007.

26- RUFFINO-NETTO A. Tuberculose: a calamidade negligenciada. Rev. Soc. Bras. Med. Trop., 35(1): 51-8, 2002.

27- SOARES PP. A dama branca e suas faces: a representação iconográfica da tuberculose. Hist. cienc. saude-Manguinhos, 1(1): 127-134, 1994.

28- SOUSA-MUÑOZ RL. (Org.). Iniciação ao Exame Clínico: Guia para o Estudante de Medicina. João Pessoa: Editora Universitária da UFPB, 2010, 168p.

29- VIEIRA AA, RIBEIRO SA. Abandono do tratamento de tuberculose utilizando-se as estratégias tratamento auto-administrado ou tratamento supervisionado no Programa Municipal de Carapicuíba, São Paulo, Brasil. J. bras. pneumol., 34(3): 159-166, 2008.

\section{Correspondência}

Pedro Henrique Perez de Moura

Av. Sinésio Guimarães 117 apt 301

João Pessoa - Paraíba - Brasil

CEP. $58.040-400$

Email.: pedrohmoura@yahoo.com 